\title{
Galois and Residuated Connections on Sets and Power Sets
}

\author{
Yong Chan Kim ${ }^{1}$ and Jung Mi Ko ${ }^{2}$ \\ Department of Mathematics, Gangneung-Wonju National University, Gangneung, 201-702, Korea
}

\begin{abstract}
We investigate the relations between various connections on set and those on power set. Moreover, we give their examples.
\end{abstract}

Key Words: Galois, dual Galois, residuated and dual residuated connections

\section{Introduction and Preliminaries}

An information consists of $(X, Y, R)$ where $X$ is a set of objects, $Y$ is a set of attributes and $R$ is a relation between $X$ and $Y$. Wille [11] introduced the formal concept lattices by allowing some uncertainty in data as examples as Galois, dual Galois, residuated and dual residuated connections. Formal concept analysis is an important mathematical tool for data analysis and knowledge processing $[1-5,8,9,11]$.

In this paper, we show that Galois, dual Galois, residuated and dual residuated connections on set induce various connections on power sets. In particular, we give their examples.

Let $X$ be a set. A relation $e_{X} \subset X \times X$ is called a partially order set (simply, poset)if it is reflexive, transitive and anti-symmetric. We can define a poset $e_{P(X)} \subset P(X) \times$ $P(X)$ as $(A, B) \in e_{P(X)}$ iff $A \subset B$ for $A, B \in P(X)$. If $\left(X, e_{X}\right)$ is a poset and we define a function $(x, y) \in e_{X}^{-1}$ iff $(y, x) \in e_{X}$, then $\left(X, e_{X}^{-1}\right)$ is a poset.

Definition 1.1. [10] Let $\left(X, e_{X}\right)$ and $\left(Y, e_{Y}\right)$ be posets and $f: X \rightarrow Y$ and $g: Y \rightarrow X$ maps.

(1) $\left(e_{X}, f, g, e_{Y}\right)$ is called a Galois connection if for all $x \in X, y \in Y,(y, f(x)) \in e_{Y}$ iff $(x, g(y)) \in e_{X}$.

(2) $\left(e_{X}, f, g, e_{Y}\right)$ is called a dual Galois connection if for all $x \in X, y \in Y,(f(x), y) \in e_{Y}$ iff $(g(y), x) \in e_{Y}$.

(3) $\left(e_{X}, f, g, e_{Y}\right)$ is called a residuated connection if for all $x \in X, y \in Y,(f(x), y) \in e_{Y}$ iff $(x, g(y)) \in e_{Y}$.

(4) $\left(e_{X}, f, g, e_{Y}\right)$ is called a dual residuated connection if for all $x \in X, y \in Y,(y, f(x)) \in e_{Y}$ iff $(g(y), x) \in e_{Y}$.

(5) $f$ is an isotone map if $\left(f\left(x_{1}\right), f\left(x_{2}\right)\right) \in e_{Y}$ for all $\left(x_{1}, x_{2}\right) \in e_{X}$.

(6) $f$ is an antitone map if $\left(f\left(x_{2}\right), f\left(x_{1}\right)\right) \in e_{Y}$ for all $\left(x_{1}, x_{2}\right) \in e_{X}$.

Theorem 1.2. [10] Let $\left(X, e_{X}\right)$ and $\left(Y, e_{Y}\right)$ be a poset and $f: X \rightarrow Y$ and $g: Y \rightarrow X$ maps.

(1) $\left(e_{X}, f, g, e_{Y}\right)$ is a Galois connection if $f, g$ are antitone maps and $(y, f(g(y))) \in e_{Y}$ and $(x, g(f(x))) \in e_{X}$.
(2) $\left(e_{X}, f, g, e_{Y}\right)$ is a dual Galois connection if $f, g$ are antitone maps and $(f(g(y)), y) \in e_{Y}$ and $(g(f(x)), x) \in$ $e_{X}$.

(3) $\left(e_{X}, f, g, e_{Y}\right)$ is a residuated connection if $f, g$ are isotone maps and $(f(g(y)), y) \in e_{Y}$ and $(x, g(f(x))) \in$ $e_{X}$.

(4) $\left(e_{X}, f, g, e_{Y}\right)$ is called a dual residuated connection if $f, g$ are isotone maps and $(y, f(g(y))) \in e_{Y}$ and $(g(f(x)), x) \in e_{X}$.

Theorem 1.3. [9] (1) $\left(e_{P(X)}, F, G, e_{P(Y)}\right)$ is a Galois connection iff $F\left(\bigcup_{i \in \Gamma} A_{i}\right)=\bigcap_{i \in \Gamma} F\left(A_{i}\right)$.

(2) $\left(e_{P(X)}, F, G, e_{P(Y)}\right)$ is a residuated connection iff $F\left(\bigcup_{i \in \Gamma} A_{i}\right)=\bigcup_{i \in \Gamma} F\left(A_{i}\right)$.

(3) $\left(e_{P(X)}, F, G, e_{P(Y)}\right)$ is a dual Galois connection iff $F\left(\bigcap_{i \in \Gamma} A_{i}\right)=\bigcup_{i \in \Gamma} F\left(A_{i}\right)$.

(4) $\left(e_{P(X)}, F, G, e_{P(Y)}\right)$ is a dual residuated connection iff $F\left(\bigcap_{i \in \Gamma} A_{i}\right)=\bigcap_{i \in \Gamma} F\left(A_{i}\right)$.

\section{Galois and Residuated Connections on Sets and Power Sets}

Theorem 2.1. Let $\left(X, e_{X}\right)$ and $\left(Y, e_{Y}\right)$ be a poset and $f: X \rightarrow Y$ and $g: Y \rightarrow X$ maps. For each $A \in P(X)$ and $B \in P(Y)$, we define operations as follows:

$$
\begin{aligned}
& F_{1}(A)=\left\{y \in Y \mid(\forall x \in X)\left(x \in A \rightarrow(f(x), y) \in e_{Y}\right)\right\}, \\
& F_{2}(A)=\left\{y \in Y \mid(\forall x \in X)\left(x \in A \rightarrow(y, f(x)) \in e_{Y}\right)\right\}, \\
& G_{1}(B)=\left\{x \in X \mid(\forall y \in Y)\left(y \in B \rightarrow(x, g(y)) \in e_{X}\right)\right\}, \\
& G_{2}(B)=\left\{x \in X \mid(\forall y \in Y)\left(y \in B \rightarrow(g(y), x) \in e_{X}\right)\right\}, \\
& H_{1}(B)=\left\{x \in X \mid(\exists y \in Y)\left(y \in B \&(x, g(y)) \in e_{X}\right)\right\}, \\
& H_{2}(B)=\left\{x \in X \mid(\exists y \in Y)\left(y \in B \&(g(y), x) \in e_{X}\right)\right\}, \\
& I_{1}(A)=\left\{y \in Y \mid(\exists x \in X)\left(x \in A \&(y, f(x)) \in e_{Y}\right)\right\}, \\
& I_{2}(A)=\left\{y \in Y \mid(\exists x \in X)\left(x \in A \&(f(x), y) \in e_{Y}\right)\right\},
\end{aligned}
$$


$\left.J_{1}(B)=\left\{x \in X \mid(\forall y \in Y)\left((x, g(y)) \in e_{X}\right) \rightarrow y \in B\right)\right\}$, $\left.J_{2}(B)=\left\{x \in X \mid(\forall y \in Y)\left((g(y), x) \in e_{X}\right) \rightarrow y \in B\right)\right\}$, $\left.K_{1}(A)=\left\{y \in Y \mid(\forall x \in X)\left((f(x), y) \in e_{Y}\right) \rightarrow x \in A\right)\right\}$, $\left.K_{2}(A)=\left\{y \in Y \mid(\forall x \in X)\left((y, f(x)) \in e_{Y}\right) \rightarrow x \in A\right)\right\}$, $L_{1}(B)=\left\{x \in X \mid(\exists y \in Y)\left(y \in B^{c} \&(x, g(y)) \in e_{X}\right)\right\}$, $L_{2}(B)=\left\{x \in X \mid(\exists y \in Y)\left(y \in B^{c} \&(g(y), x) \in e_{X}\right)\right\}$, $M_{1}(A)=\left\{y \in Y \mid(\exists x \in X)\left(x \in A^{c} \&(y, f(x)) \in e_{Y}\right)\right\}$, $M_{2}(A)=\left\{y \in Y \mid(\exists x \in X)\left(x \in A^{c} \&(f(x), y) \in e_{Y}\right)\right\}$,

Then the following statements hold:

(1) $F_{1}(\{x\})=I_{2}(\{x\})=M_{2}\left((\{x\})^{c}\right)=\left(e_{Y}\right)_{f(x)}$, $F_{2}(\{x\})=I_{1}(\{x\})=M_{1}\left((\{x\})^{c}\right)=\left(e_{Y}\right)_{f(x)}^{-1}$, $K_{2}\left((\{x\})^{c}\right)=\left(\left(e_{Y}\right)_{f(x)}^{-1}\right)^{c}, K_{1}\left(\{x\}^{c}\right)=\left(e_{Y}\right)_{f(x)}^{c}$.

(2) $G_{1}(\{y\})=H_{1}(\{y\})=L_{1}\left(\{y\}^{c}\right)=\left(e_{X}\right)_{g(y)}^{-1}$, $G_{2}(\{y\})=H_{2}(\{y\})=L_{2}\left(\{y\}^{c}\right)=\left(e_{X}\right)_{g(y)}$. $J_{1}\left((\{y\})^{c}\right)=\left(\left(e_{X}\right)_{g(y)}^{-1}\right)^{c}, J_{2}\left(\{y\}^{c}\right)=\left(e_{X}\right)_{g(y)}^{c}$.

(3) $\left(e_{X}, f, g, e_{Y}\right)$ is a Galois connection iff $\left(e_{P(X)}, F_{2}, G_{1}, e_{P(Y)}\right)$ is a Galois connection iff $\left(e_{P(X)}, K_{2}, H_{1}, e_{P(Y)}\right)$ is a dual residuated connection iff $\left(e_{P(X)}, M_{1}, L_{1}, e_{P(Y)}\right)$ is a dual Galois connection iff $\left(e_{P(X)}, I_{1}, J_{1}, e_{P(Y)}\right)$ is a residuated connection.

(4) $\left(e_{X}, f, g, e_{Y}\right)$ is a residuated connection iff $\left(e_{P(X)}, F_{1}, G_{1}, e_{P(Y)}\right)$ is a Galois connection iff $\left(e_{P(X)}, K_{1}, H_{1}, e_{P(Y)}\right)$ is a dual residuated connection iff $\left(e_{P(X)}, M_{2}, L_{1}, e_{P(Y)}\right)$ is a dual Galois connection iff $\left(e_{P(X)}, I_{2}, J_{1}, e_{P(Y)}\right)$ is a residuated connection.

(5) $\left(e_{X}, f, g, e_{Y}\right)$ is a dual Galois connection iff $\left(e_{P(X)}, F_{1}, G_{2}, e_{P(Y)}\right)$ is a Galois connection iff $\left(e_{P(X)}, K_{1}, H_{2}, e_{P(Y)}\right)$ is a dual residuated connection iff $\left(e_{P(X)}, M_{2}, L_{2}, e_{P(Y)}\right)$ is a dual Galois connection iff $\left(e_{P(X)}, I_{2}, J_{2}, e_{P(Y)}\right)$ is a residuated connection.

(6) $\left(e_{X}, f, g, e_{Y}\right)$ is a dual residuated connection iff $\left(e_{P(X)}, F_{2}, G_{2}, e_{P(Y)}\right)$ is a Galois connection iff $\left(e_{P(X)}, K_{2}, H_{2}, e_{P(Y)}\right)$ is a dual residuated connection iff $\left(e_{P(X)}, M_{1}, L_{2}, e_{P(Y)}\right)$ is a dual Galois connection iff $\left(e_{P(X)}, I_{1}, J_{2}, e_{P(Y)}\right)$ is a residuated connection.

(7) If $f\left(\vee_{i \in \Gamma} x_{i}\right)=\wedge_{i \in \Gamma} f\left(x_{i}\right)$ for $x_{i} \in X$, there exists a function $g: Y \rightarrow X$ such that $\left(e_{X}, f, g, e_{Y}\right)$ is a Galois connection. Moreover, $\left(e_{P(X)}, F_{2}, G_{1}, e_{P(Y)}\right)$ is a Galois connection, $\left(e_{P(X)}, K_{2}, H_{1}, e_{P(Y)}\right)$ is a dual residuated connection, $\left(e_{P(X)}, M_{1}, L_{1}, e_{P(Y)}\right)$ is a dual Galois connection and $\left(e_{P(X)}, I_{1}, J_{1}, e_{P(Y)}\right)$ is a residuated connection.

(8) If $f\left(\vee_{i \in \Gamma} x_{i}\right)=\vee_{i \in \Gamma} f\left(x_{i}\right)$ for $x_{i} \in X$, there exists a function $g: Y \rightarrow X$ such that a residuated connection. Moreover, $\left(e_{P(X)}, F_{1}, G_{1}, e_{P(Y)}\right)$ is a Galois connection, $\left(e_{P(X)}, K_{1}, H_{1}, e_{P(Y)}\right)$ is a dual residuated connection, $\left(e_{P(X)}, M_{2}, L_{1}, e_{P(Y)}\right)$ is a dual Galois connection and $\left(e_{P(X)}, I_{2}, J_{1}, e_{P(Y)}\right)$ is a residuated connection.

(9) If $f\left(\wedge_{i \in \Gamma} x_{i}\right)=\vee_{i \in \Gamma} f\left(x_{i}\right)$ for $x_{i} \in X$, there exists a function $g: Y \rightarrow X$ such that $\left(e_{X}, f, g, e_{Y}\right)$ is a dual
Galois connection. Moreover, $\left(e_{P(X)}, F_{1}, G_{2}, e_{P(Y)}\right)$ is a Galois connection, $\left(e_{P(X)}, K_{1}, H_{2}, e_{P(Y)}\right)$ is a dual residuated connection, $\left(e_{P(X)}, M_{2}, L_{2}, e_{P(Y)}\right)$ is a dual Galois connection and $\left(e_{P(X)}, I_{2}, J_{2}, e_{P(Y)}\right)$ is a residuated connection.

(10) If $f\left(\wedge_{i \in \Gamma} x_{i}\right)=\wedge_{i \in \Gamma} f\left(x_{i}\right)$ for $x_{i} \in X$, there exists a function $g: Y \rightarrow X$ such that $\left(e_{X}, f, g, e_{Y}\right)$ is a dual residuated connection. Moreover, $\left(e_{P(X)}, F_{2}, G_{2}, e_{P(Y)}\right)$ is a Galois connection and $\left(e_{P(X)}, K_{2}, H_{2}, e_{P(Y)}\right)$ is a dual residuated connection, $\left(e_{P(X)}, M_{1}, L_{2}, e_{P(Y)}\right)$ is a dual Galois connection and $\left(e_{P(X)}, I_{1}, J_{2}, e_{P(Y)}\right)$ is a residuated connection.

Proof. (1) $y \in F_{1}(\{x\})$ iff $(\forall z \in X)(z \in\{x\} \rightarrow$ $\left.(f(z), y) \in e_{Y}\right)$ iff $(f(x), y) \in e_{Y}$. Similarly, $F_{2}(\{x\})=$ $\left(e_{Y}\right)_{f(x)}^{-1}$. We have $K_{1}\left(\{x\}^{c}\right)=\left(e_{Y}\right)_{f(x)}^{c}$ from: $y \in$ $K_{1}\left(\{x\}^{c}\right)$ iff $(\forall z \in X)\left((f(z), y) \in e_{Y} \rightarrow z \in\{x\}^{c}\right)$ iff $(\forall z \in X)\left(z \in\{x\} \rightarrow(f(z), y) \notin e_{Y}\right)$ iff $(f(x), y) \notin e_{Y}$. Similarly, $K_{2}\left((\{x\})^{c}\right)=\left(\left(e_{Y}\right)_{f(x)}^{-1}\right)^{c}$.

Other cases and (2) are similarly proved as in (1).

(3) First, if $(x, g(y)) \in e_{X}$ iff $(y, f(x)) \in e_{Y}$, then $\left(A, G_{1}(B)\right) \in e_{P(X)}$ iff $\left(B, F_{2}(A)\right) \in e_{P(Y)}$.

$$
\begin{aligned}
& \left(B, F_{2}(A)\right) \in e_{P(Y)} \\
& \text { iff }(\forall y \in Y)\left((y \in B) \rightarrow y \in F_{2}(A)\right) \\
& \text { iff }(\forall y \in Y)((y \in B) \rightarrow(\forall x \in X)(x \in A \\
& \left.\left.\quad \rightarrow(y, f(x)) \in e_{Y}\right)\right) \\
& \text { iff }(\forall y \in Y)(\forall x \in X)(x \in A \rightarrow(y \in B \\
& \left.\left.\quad \rightarrow(x, g(y)) \in e_{X}\right)\right) \\
& \text { iff }(\forall x \in X)(x \in A \rightarrow(\forall y \in Y)(y \in B \\
& \left.\left.\quad \rightarrow(x, g(y)) \in e_{X}\right)\right) \\
& \text { iff }(\forall x \in X)\left(x \in A \rightarrow x \in G_{1}(B)\right) \\
& \text { iff }\left(A, G_{1}(B)\right) \in e_{P(X) .}
\end{aligned}
$$

Conversely, put $A=\{x\}$ and $B=\{y\}$. Since $F_{2}(\{x\})=\left(e_{Y}\right)_{f(x)}^{-1}$ and $G_{1}(\{y\})=\left(e_{X}\right)_{g(y)}^{-1}$ from (1) and (2), we have

$$
\begin{aligned}
& (y, f(x)) \in e_{Y} \text { iff }\left(\{y\}, F_{2}(\{x\})\right) \in e_{P(Y)} \\
& \text { iff }\left(\{x\}, G_{1}(\{y\})\right) \in e_{P(X)} \text { iff }(x, g(y)) \in e_{X} .
\end{aligned}
$$

Second, if $(x, g(y)) \in e_{X}$ iff $(y, f(x)) \in e_{Y}$, then $\left(H_{1}(B), A\right) \in e_{P(X)}$ iff $\left(B, K_{2}(A)\right) \in e_{P(Y)}$. 


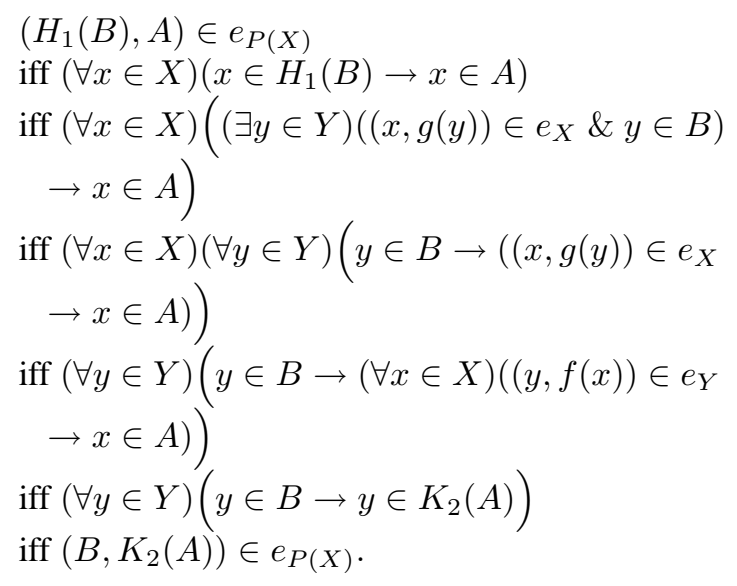

Put $A=\{x\}^{c}$ and $B=\{y\}$. Since $K_{2}\left(\{x\}^{c}\right)=$ $\left(\left(e_{Y}\right)_{f(x)}^{-1}\right)^{c}$ and $H_{1}(\{y\})=\left(e_{X}\right)_{g(y)}^{-1}$ from (1) and (2), we have

$(x, g(y)) \notin e_{X}$ iff $\left(H_{1}(\{y\}),\{x\}^{c}\right) \in e_{P(X)}$ iff $\left(\{y\}, K_{2}\left(\{x\}^{c}\right)\right) \in e_{P(Y)}$ iff $(y, f(x)) \notin e_{Y}$.

Third, if $(x, g(y)) \in e_{X}$ iff $(y, f(x)) \in e_{Y}$, then $\left(L_{1}(B), A\right) \in e_{P(X)}$ iff $\left(M_{1}(A), B\right) \in e_{P(Y)}$.

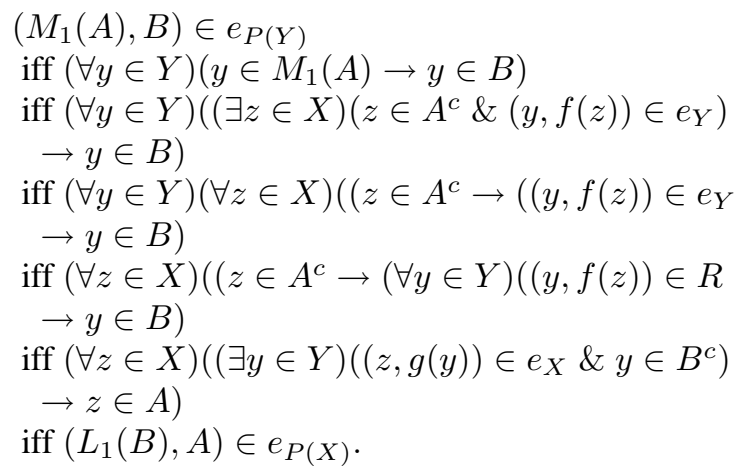

Put $A=\{x\}^{c}$ and $B=\{y\}^{c}$. Since $M_{1}\left(\{x\}^{c}\right)=$ $\left(e_{Y}\right)_{f(x)}^{-1}$ and $L_{1}\left(\{y\}^{c}\right)=\left(e_{X}\right)_{g(y)}^{-1}$ from (1) and (2), we have

$\left(y, f(x) \notin e_{Y}\right.$ iff $y \in M_{1}\left(\{x\}^{c}\right)^{c}$ iff

$\left(M_{1}\left(\{x\}^{c}\right),\{y\}^{c}\right) \in e_{P(Y)}$ iff $\left(L_{1}\left(\{y\}^{c}\right),\{x\}^{c}\right) \in e_{P(X)}$

iff $x \in L_{1}\left(\{y\}^{c}\right)^{c}$ iff $\left(x, g(y) \notin e_{X}\right.$.

Forth, if $(x, g(y)) \in e_{X}$ iff $(y, f(x)) \in e_{Y}$, then

$$
\left(I_{1}(A), B\right) \in e_{P(Y)} \text { iff }\left(A, J_{1}(B)\right) \in e_{P(X)} .
$$

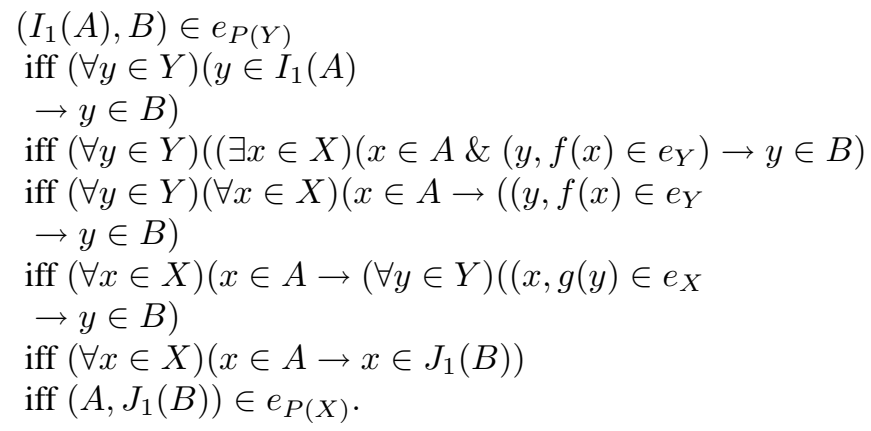

Put $A=\{x\}$ and $B=\{y\}^{c}$. Since $I_{1}(\{x\})=$ $\left(e_{Y}\right)_{f(x)}^{-1}$ and $J_{1}\left(\{y\}^{c}\right)=\left(\left(e_{X}\right)_{g(y)}^{-1}\right)^{c}$ from (1) and (2), we have

$\left(y, f(x) \notin e_{Y}\right.$ iff $y \in I_{1}(\{x\})^{c}$ iff

$\left(I_{1}(\{x\}),\{y\}^{c}\right) \in e_{P(Y)}$ iff $\left(\{x\}, J_{1}\left(\{y\}^{c}\right)\right) \in e_{P(X)}$

iff $x \in J_{1}\left(\{y\}^{c}\right)$ iff $(x, g(y)) \notin e_{X}$.

(4) Let $(x, g(y)) \in e_{X}$ iff $(f(x), y) \in e_{Y}$ be given. Then $\left(A, G_{1}(B)\right) \in e_{P(X)}$ iff $\left(B, F_{1}(A)\right) \in e_{P(Y)}$ from:

$$
\begin{aligned}
& \left(B, F_{1}(A)\right) \in e_{P(Y)} \\
& \text { iff }(\forall y \in Y)\left((y \in B) \rightarrow y \in F_{1}(A)\right) \\
& \text { iff }(\forall y \in Y)((y \in B) \rightarrow(\forall x \in X)(x \in A \\
& \left.\left.\quad \rightarrow(f(x), y) \in e_{Y}\right)\right) \\
& \text { iff }(\forall y \in Y)(\forall x \in X)(x \in A \rightarrow(y \in B \\
& \left.\left.\quad \rightarrow(x, g(y)) \in e_{X}\right)\right) \\
& \text { iff }(\forall x \in X)(x \in A \rightarrow(\forall y \in Y)(y \in B \\
& \left.\left.\quad \rightarrow(x, g(y)) \in e_{X}\right)\right) \\
& \text { iff }(\forall x \in X)\left(x \in A \rightarrow x \in G_{1}(B)\right) \\
& \text { iff }\left(A, G_{1}(B)\right) \in e_{P(X) .}
\end{aligned}
$$

Conversely, put $A=\{x\}$ and $B=\{y\}$. Since $F_{1}(\{x\})=$ $\left(e_{Y}\right)_{f(x)}$ and $G_{1}(\{y\})=\left(e_{X}\right)_{g(y)}^{-1}$ from (1) and (2), we have

$$
(f(x), y) \in e_{Y} \text { iff } y \in F_{1}(\{x\})
$$

iff $\left(\{y\}, F_{1}(\{x\})\right) \in e_{P(Y)}$ iff $\left(\{x\}, G_{1}(\{y\})\right) \in e_{P(X)}$

$$
\text { iff } x \in G_{1}(\{x\}) \text { iff }(x, g(y)) \in e_{X} \text {. }
$$

Second, if $(x, g(y)) \in e_{X}$ iff $(f(x), y) \in e_{Y}$, then 
$\left(H_{1}(B), A\right) \in e_{P(X)}$ iff $\left(B, K_{1}(A)\right) \in e_{P(Y)}$ from:

$\left(H_{1}(B), A\right) \in e_{P(X)}$

iff $(\forall x \in X)\left(x \in H_{1}(B) \rightarrow x \in A\right)$

iff $(\forall x \in X)\left((\exists y \in Y)\left((x, g(y)) \in e_{X} \& y \in B\right)\right.$

$\rightarrow x \in A)$

iff $(\forall x \in X)(\forall y \in Y)\left(y \in B \rightarrow\left((x, g(y)) \in e_{X}\right.\right.$

$\rightarrow x \in A)$ )

iff $(\forall y \in Y)\left(y \in B \rightarrow(\forall x \in X)\left((f(x), y) \in e_{Y}\right.\right.$

$\rightarrow x \in A))$

iff $(\forall y \in Y)\left(y \in B \rightarrow y \in K_{1}(A)\right)$

iff $\left(B, K_{1}(A)\right) \in e_{P(Y)}$.

Put $A=\{x\}^{c}$ and $B=\{y\}$. Since $K_{1}\left(\{x\}^{c}\right)=$ $\left(e_{Y}\right)_{f(x)}^{c}$ and $H_{1}(\{y\})=\left(e_{X}\right)_{g(y)}^{-1}$ from (1) and (2), we have

$$
(x, g(y)) \notin e_{X} \text { iff } x \notin H_{1}(\{y\})
$$

iff $\left(H_{1}\left(\{y\},\{x\}^{c}\right) \in e_{P(X)}\right.$ iff $\left(\{y\}, K_{1}\left(\{x\}^{c}\right)\right) \in e_{P(Y)}$

iff $y \in K_{1}(\{x\})$ iff $(f(x), y) \notin e_{Y}$.

Third, if $(x, g(y)) \in e_{X}$ iff $(f(x), y) \in e_{Y}$, then $\left(L_{1}(B), A\right) \in e_{P(X)}$ iff $\left(M_{2}(A), B\right) \in e_{P(Y)}$.

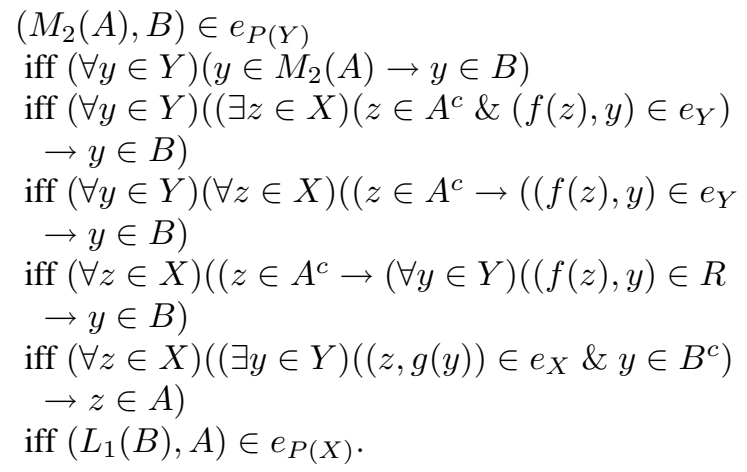

Put $A=\{x\}^{c}$ and $B=\{y\}^{c}$. Since $M_{2}\left(\{x\}^{c}\right)=$ $\left(e_{Y}\right)_{f(x)}$ and $L_{1}\left(\{y\}^{c}\right)=\left(e_{X}\right)_{g(y)}^{-1}$ from (1) and (2), we have

$(f(x), y) \notin e_{Y}$ iff $y \in M_{2}\left(\{x\}^{c}\right)^{c}$ iff

$\left(M_{2}\left(\{x\}^{c}\right),\{y\}^{c}\right) \in e_{P(Y)}$ iff $\left(L_{1}\left(\{y\}^{c}\right),\{x\}^{c}\right) \in e_{P(X)}$ iff $x \in L_{1}\left(\{y\}^{c}\right)^{c}$ iff $\left(x, g(y) \notin e_{X}\right.$.

Forth, if $(x, g(y)) \in e_{X}$ iff $(f(x), y) \in e_{Y}$, then $\left(I_{2}(A), B\right) \in e_{P(Y)}$ iff $\left(A, J_{1}(B)\right) \in e_{P(X)}$.

$\left(I_{2}(A), B\right) \in e_{P(Y)}$

iff $(\forall y \in Y)\left(y \in I_{2}(A) \rightarrow y \in B\right)$

iff $(\forall y \in Y)\left((\exists x \in X)\left(x \in A \&(f(x), y) \in e_{Y}\right) \rightarrow y \in B\right)$

iff $(\forall y \in Y)(\forall x \in X)\left(x \in A \rightarrow\left((f(x), y) \in e_{Y} \rightarrow y \in B\right)\right.$

iff $(\forall x \in X)\left(x \in A \rightarrow(\forall y \in Y)\left(\left(x, g(y) \in e_{X} \rightarrow y \in B\right)\right.\right.$

iff $(\forall x \in X)\left(x \in A \rightarrow x \in J_{1}(B)\right)$

iff $\left(A, J_{1}(B)\right) \in e_{P(X)}$.
Put $A=\{x\}$ and $B=\{y\}^{c}$. Since $I_{2}(\{x\})=$ $\left(e_{Y}\right)_{f(x)}$ and $J_{1}\left(\{y\}^{c}\right)=\left(\left(e_{X}\right)_{g(y)}^{-1}\right)^{c}$ from (1) and (2), we have

$(f(x), y) \notin e_{Y}$ iff $y \in I_{1}(\{x\})^{c}$ iff

$\left(I_{1}(\{x\}),\{y\}^{c}\right) \in e_{P(Y)}$ iff $\left(\{x\}, J_{1}\left(\{y\}^{c}\right)\right) \in e_{P(X)}$

iff $x \in J_{1}\left(\{y\}^{c}\right)$ iff $(x, g(y)) \notin e_{X}$.

(5) and (6) are similarly proved as in (3) and (4).

(7) Define $g(y)=\bigvee\{x \mid y \leq f(x)\}$. If $y \leq f(x)$, then $x \leq g(y)$. If $x \leq g(y)$, then $f(x) \geq f(g(y))=f(\vee x)=$ $\wedge f(x) \geq y$. Hence $\left(e_{X}, f, g, e_{Y}\right)$ is a Galois connection. Others follow (3).

(8) Define $g(y)=\bigvee\{x \mid f(x) \leq y\}$. If $f(x) \leq y$, then $x \leq g(y)$. If $x \leq g(y)$, then $f(x) \leq f(g(y))=$ $f(\vee x)=\vee f(x) \leq y$. Hence $\left(e_{X}, f, g, e_{Y}\right)$ is a residuated connection. Others follow (4).

(9) Define $g(y)=\bigwedge\{x \mid f(x) \leq y\}$. If $f(x) \leq y$, then $g(y) \leq x$. If $g(y) \leq x$, then $f(x) \leq f(g(y))=f(\wedge x)=$ $\vee f(x) \leq y$. Hence $\left(e_{X}, f, g, e_{Y}\right)$ is a dual Galois connection. Others follow (5).

(10) Define $g(y)=\bigwedge\{x \mid y \leq f(x)\}$. If $y \leq f(x)$, then $g(y) \leq x$. If $g(y) \leq x$, then $f(x) \geq f(g(y))=$ $f(\wedge x)=\wedge f(x) \geq y$. Hence $\left(e_{X}, f, g, e_{Y}\right)$ is a dual residuated connection. Others follow (6).

Example 2.2. Let $\left(X=\{a, b, c, d\}, e_{X}\right)$ and $(Y=$ $\left.\{x, y, z\}, e_{Y}\right)$ be a poset with

$$
\begin{gathered}
e_{X}=\{(a, a),(a, b),(a, c),(a, d),(b, b),(b, d), \\
(c, c),(c, d),(d, d)\} . \\
e_{Y}=\{(x, x),(x, y),(x, z),(y, y),(z, y),(z, z)\} .
\end{gathered}
$$

(1) Let $f: X \rightarrow Y$ as $f(a)=x, f(b)=z, f(c)=$ $f(d)=y$. Then $f\left(\vee_{i \in \Gamma} x_{i}\right)=\vee_{i \in \Gamma} f\left(x_{i}\right)$ for $x_{i} \in X$. Define $g(w)=\bigvee\{s \in X \mid f(s) \leq w\}$. Then $g: Y \rightarrow X$ as $g(x)=a, g(y)=d, g(z)=b$. Then $\left(e_{X}, f, g, e_{Y}\right)$ is a residuated connection.

By Theorem 2.1 (1) and (2), we obtain

$$
\begin{aligned}
& \left(F_{1}=I_{2}\right)(\{a\})=M_{2}\left(\{a\}^{c}\right)=\{x, y, z\}=\left(e_{Y}\right)_{f(a)}, \\
& \left(F_{1}=I_{2}\right)(\{b\})=M_{2}\left(\{a\}^{c}\right)=\{y, z\}=\left(e_{Y}\right)_{f(b)}, \\
& \left(F_{1}=I_{2}\right)(\{c\})=M_{2}\left(\{a\}^{c}\right)=\{y\}=\left(e_{Y}\right)_{f(c)}, \\
& \left(F_{1}=I_{2}\right)(\{d\})=M_{2}\left(\{a\}^{c}\right)=\{y\}=\left(e_{Y}\right)_{f(d)} .
\end{aligned}
$$

$$
\begin{aligned}
K_{1}\left(\{a\}^{c}\right) & =\emptyset=\left(e_{Y}\right)_{f(a)}^{c}, \\
K_{1}\left(\{b\}^{c}\right) & =\{x\}=\left(e_{Y}\right)_{f(b)}^{c}, \\
K_{1}\left(\{c\}^{c}\right) & =\{x, z\}=\left(e_{Y}\right)_{f(c)}^{c}, \\
K_{1}\left(\{d\}^{c}\right) & =\{x, z\}=\left(e_{Y}\right)_{f(d)}^{c} .
\end{aligned}
$$




$$
\begin{aligned}
\left(G_{1}=H_{1}\right)(\{x\}) & =L_{1}\left(\{x\}^{c}\right)=\{a\}=\left(e_{X}\right)_{g(x)}^{-1}, \\
\left(G_{1}=H_{1}\right)(\{y\}) & =L_{1}\left(\{y\}^{c}\right)=X=\left(e_{Y}\right)_{g(y)}^{-1}, \\
\left(G_{1}=\right. & \left.H_{1}\right)(\{z\})=L_{1}\left(\{z\}^{c}\right)=\{a, b\}=\left(e_{Y}\right)_{g(z)}^{-1} . \\
J_{1}\left(\{x\}^{c}\right) & =\{b, c, d\}=\left(\left(e_{X}\right)_{g(x)}^{-1}\right)^{c}, \\
J_{1}\left(\{y\}^{c}\right) & =\emptyset=\left(\left(e_{Y}\right)_{g(y)}^{-1}\right)^{c}, \\
J_{1}\left(\{z\}^{c}\right) & =\{c, d\}=\left(\left(e_{Y}\right)_{g(z)}^{-1}\right)^{c} .
\end{aligned}
$$

We obtain $F_{1}: P(X) \rightarrow P(Y)$ and $G_{1}: P(Y) \rightarrow$ $P(X)$ as follows:

$$
\begin{gathered}
F_{1}(A)= \begin{cases}Y & \text { if } A \in\{\emptyset,\{a\}\}, \\
\{y, z\} & \text { if } A \in\{\{b\},\{a, b\}\}, \\
\{y\} & \text { otherwise, }\end{cases} \\
G_{1}(B)= \begin{cases}X & \text { if } B \in\{\emptyset,\{y\}\}, \\
\{a, b\} & \text { if } B \in\{\{z\},\{y, z\}\}, \\
\{a\} & \text { otherwise. }\end{cases}
\end{gathered}
$$

Then $\left(e_{P(X)}, F_{1}, G_{1}, e_{P(Y)}\right)$ is a Galois connection.

We obtain $K_{1}: P(X) \rightarrow P(Y)$ and $H_{1}: P(Y) \rightarrow$ $P(X)$ as follows:

$$
\begin{gathered}
K_{1}(A)= \begin{cases}Y & \text { if } A=X, \\
\{x\} & \text { if } a \in A,\{a, b\} \not \subset A, \\
\{x, z\} & \text { if }\{a, b\} \subset A \neq X, \\
\emptyset & \text { otherwise, }\end{cases} \\
H_{1}(B)= \begin{cases}\emptyset & \text { if } B=\emptyset, \\
X & \text { if } y \in B, \\
\{a, b\} & \text { if } z \in B, y \notin B, \\
\{a\} & \text { otherwise. }\end{cases}
\end{gathered}
$$

Then $\left(e_{P(X)}, K_{1}, H_{1}, e_{P(Y)}\right)$ is a dual residuated connection.

We obtain $M_{2}: P(X) \rightarrow P(Y)$ and $L_{1}: P(Y) \rightarrow$ $P(X)$ as follows:

$$
\begin{gathered}
M_{2}(A)= \begin{cases}\emptyset & \text { if } A=X, \\
\{y\} & \text { if } A \in\{\{a, b\},\{a, b, c\},\{a, b, d\}\}, \\
\{y, z\} & \text { if } A \in\{\{a\},\{a, d\},\{a, c, d\}\}, \\
Y & \text { otherwise, }\end{cases} \\
L_{1}(B)= \begin{cases}\emptyset & \text { if } B=X, \\
\{a\} & \text { if } B=\{y, z\}, \\
\{a, b\} & \text { if } B \in\{\{y\},\{x, y\}, \\
X & \text { otherwise. }\end{cases}
\end{gathered}
$$

Then $\left(e_{P(X)}, M_{2}, L_{1}, e_{P(Y)}\right)$ is a dual Galois connection.

We obtain $I_{2}: P(X) \rightarrow P(Y)$ and $J_{1}: P(Y) \rightarrow$ $P(X)$ as follows:

$$
I_{2}(A)= \begin{cases}\emptyset & \text { if } A=\emptyset \\ \{z\} & \text { if } A \in\{\{c\},\{d\},\{c, d\}\}, \\ \{y, z\} & \text { if } A \in\{\{b\},\{b, c\},\{b, d\},\{b, c, d\}\} \\ Y & \text { otherwise, }\end{cases}
$$

$$
J_{1}(B)= \begin{cases}X & \text { if } B=Y \\ \{b, c, d\} & \text { if } B=\{y, z\} \\ \{c, d\} & \text { if } B \in\{\{y\},\{x, y\}\} \\ \emptyset & \text { otherwise. }\end{cases}
$$

Then $\left(e_{P(X)}, I_{2}, J_{1}, e_{P(Y)}\right)$ is a residuated connection.

(2) Let $f: X \rightarrow Y$ as $f(a)=y, f(b)=z, f(c)=$ $f(d)=x$. Then $f\left(\vee_{i \in \Gamma} x_{i}\right)=\wedge_{i \in \Gamma} f\left(x_{i}\right)$ for $x_{i} \in X$. Define $g(w)=\bigvee\{s \in X \mid f(s) \geq w\}$. Then $g: Y \rightarrow X$ as $g(x)=d, g(y)=a, g(z)=b$. Then $\left(e_{X}, f, g, e_{Y}\right)$ is a Galois connection.

By Theorem 2.1 (1) and (2), we obtain

$$
\begin{aligned}
&\left(F_{2}=I_{1}\right)(\{a\})=M_{1}\left(\{a\}^{c}\right)=Y=\left(e_{Y}\right)_{f(a)}^{-1}, \\
&\left(F_{2}=I_{1}\right)(\{b\})= M_{1}\left(\{b\}^{c}\right)=\{x, z\}=\left(e_{Y}\right)_{f(b)}^{-1}, \\
&\left(F_{2}=I_{1}\right)(\{c\})=M_{1}\left(\{c\}^{c}\right)=\{x\}=\left(e_{Y}\right)_{f(c)}^{-1}, \\
&\left(F_{2}=I_{1}\right)(\{d\})=M_{1}\left(\{d\}^{c}\right)=\{x\}=\left(e_{Y}\right)_{f(d)}^{-1} . \\
&\left(G_{1}=H_{1}\right)(\{x\})=L_{1}\left(\{x\}^{c}\right)=X=\left(e_{X}\right)_{g(x)}^{-1}, \\
&\left(G_{1}=H_{1}\right)(\{y\})=L_{1}\left(\{y\}^{c}\right)=\{a\}=\left(e_{Y}\right)_{g(y)}^{-1}, \\
&\left(G_{1}=H_{1}\right)(\{z\})=L_{1}\left(\{z\}^{c}\right)=\{a, b\}=\left(e_{Y}\right)_{g(z)}^{-1} . \\
& J_{1}\left(\{x\}^{c}\right)=\emptyset=\left(\left(e_{X}\right)_{g(x)}^{-1}\right)^{c}, \\
& J_{1}\left(\{y\}^{c}\right)=\{b, c, d\}=\left(\left(e_{Y}\right)_{g(y)}^{-1}\right)^{c}, \\
& J_{1}\left(\{z\}^{c}\right)=\{c, d\}=\left(\left(e_{Y}\right)_{g(z)}^{-1}\right)^{c} . \\
& K_{2}\left(\{a\}^{c}\right)=\emptyset=\left(e_{Y}^{-1}\right)_{f(a)}^{c}, \\
& K_{2}\left(\{b\}^{c}\right)=\{y\}=\left(e_{Y}^{-1}\right)_{f(b)}^{c}, \\
& K_{2}\left(\{c\}^{c}\right)=\{y, z\}=\left(e_{Y}^{-1}\right)_{f(c)}^{c}, \\
& K_{2}\left(\{d\}^{c}\right)=\{y, z\}=\left(e_{Y}^{-1}\right)_{f(d)}^{c} .
\end{aligned}
$$

We obtain $F_{2}: P(X) \rightarrow P(Y)$ and $G_{1}: P(Y) \rightarrow$ $P(X)$ as follows:

$$
\begin{gathered}
F_{2}(A)= \begin{cases}Y & \text { if } A \in\{\emptyset,\{a\}\}, \\
\{x, z\} & \text { if } A \in\{\{b\},\{a, b\}\}, \\
\{x\} & \text { otherwise, }\end{cases} \\
G_{1}(B)= \begin{cases}X & \text { if } B \in\{\emptyset,\{x\}\}, \\
\{a, b\} & \text { if } B \in\{\{z\},\{x, z\}\}, \\
\{a\} & \text { otherwise. }\end{cases}
\end{gathered}
$$

Then $\left(e_{P(X)}, F_{2}, G_{1}, e_{P(Y)}\right)$ is a Galois connection.

We obtain $K_{2}: P(X) \rightarrow P(Y)$ and $H_{1}: P(Y) \rightarrow$ $P(X)$ as follows:

$$
\begin{gathered}
K_{2}(A)= \begin{cases}Y & \text { if } A=X, \\
\{y\} & \text { if } a \in A,\{a, b\} \not \subset A, \\
\{y, z\} & \text { if }\{a, b\} \subset A \neq X, \\
\emptyset & \text { otherwise, }\end{cases} \\
H_{1}(B)= \begin{cases}\emptyset & \text { if } B=\emptyset, \\
X & \text { if } x \in B, \\
\{a, b\} & \text { if } z \in B, x \notin B, \\
\{a\} & \text { otherwise. }\end{cases}
\end{gathered}
$$

Then $\left(e_{P(X)}, K_{2}, H_{1}, e_{P(Y)}\right)$ is a dual residuated connection. 
We obtain $M_{1}: P(X) \rightarrow P(Y)$ and $L_{1}: P(Y) \rightarrow$ $P(X)$ as follows:

$$
M_{1}(A)= \begin{cases}\emptyset & \text { if } A=X \\ \{x\} & \text { if } A \in\{\{a, b\},\{a, b, c\},\{a, b, d\}\} \\ \{x, z\} & \text { if } A \in\{\{a\},\{a, c\},\{a, d\},\{a, c, d\}\} \\ Y & \text { otherwise }\end{cases}
$$

$$
L_{1}(B)= \begin{cases}\emptyset & \text { if } B=Y \\ \{a\} & \text { if } B=\{x, z\} \\ \{a, b\} & \text { if } B \in\{\{x\},\{x, y\}\} \\ X & \text { otherwise. }\end{cases}
$$

Then $\left(e_{P(X)}, M_{1}, L_{1}, e_{P(Y)}\right)$ is a dual Galois connection.

We obtain $I_{1}: P(X) \rightarrow P(Y)$ and $J_{1}: P(Y) \rightarrow$ $P(X)$ as follows:

$$
\begin{gathered}
I_{1}(A)= \begin{cases}\emptyset & \text { if } A=\emptyset, \\
\{z\} & \text { if } A \in\{\{c\},\{d\},\{c, d\}\}, \\
\{x, z\} & \text { if } A \in\{\{b\},\{b, c\},\{b, d\},\{b, c, d\}\}, \\
Y & \text { otherwise, }\end{cases} \\
J_{1}(B)= \begin{cases}X & \text { if } B=Y, \\
\{b, c, d\} & \text { if } B=\{x, z\}, \\
\{b, d\} & \text { if } B \in\{\{x\},\{x, y\}\}, \\
\emptyset & \text { otherwise. }\end{cases}
\end{gathered}
$$

Then $\left(e_{P(X)}, I_{1}, J_{1}, e_{P(Y)}\right)$ is a residuated connection.

\section{References}

[1] R. Bělohlávek, "Similarity relations in concept lattices," J. Logic and Computation, vol. 10, no 6,pp.823-845, 2000.

[2] R. Bělohlávek, "Lattices of fixed points of Galois connections," Math. Logic Quart., vol. 47, pp.111-116, 2001.

[3] R. Bělohlávek, "Concept lattices and order in fuzzy logic," Ann. Pure Appl. Logic, vol. 128, pp.277-298, 2004.

[4] R. Bělohlávek, Fuzzy relational systems, Kluwer Academic Publisher, New York, 2002.
[5] G. Georgescu, A. Popescue," Non-dual fuzzy connections," Arch. Math. Log., vol. 43, pp. 1009-1039, 2004.

[6] J.G. Garcia, I.M. Perez, M.A.P. Vicente, D. Zhang," Fuzzy Galois connections categorically," Math. Log. Quart., vol. 56, pp. 131-147, 2010.

[7] H. Lai, D. Zhang," Concept lattices of fuzzy contexts: Formal concept analysis vs. rough set theory," Int. J. Approx. Reasoning, vol. 50, pp.695-707, 2009.

[8] Y.C. Kim, J.W. Park, "Join preserving maps and various concepts," Int.J. Contemp. Math. Sciences, vol. 5, no.5, pp.243-251, 2010.

[9] J.M. Ko, Y.C. Kim, "Various connections and their relations," submit to Int. J. Fuzzy Logic and Intelligent Systems.

[10] Ewa. Orlowska, I. Rewitzky, "Algebras for Galoisstyle connections and their discrete duality," Fuzzy Sets and Systems, vol.161, pp.1325-1342, 2010.

[11] R. Wille, Restructuring lattice theory; an approach based on hierarchies of concept, in: 1. Rival(Ed.), Ordered Sets, Reidel, Dordrecht, Boston, 1982.

[12] Wei Yao, Ling-Xia Lu," Fuzzy Galois connections on fuzzy posets," Math. Log. Quart., vol. 55, pp. 105112, 2009.

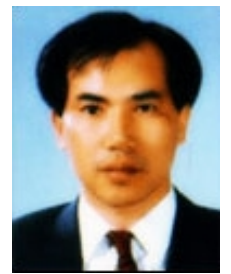

\section{Yong Chan Kim}

He received the M.S and Ph.D. degrees in Department of Mathematics from Yonsei University, in 1984 and 1991, respectively. From 1991 to present, he is a professor in the Department of Mathematics, Gangneung -Wonju University. His research interests are fuzzy topology and fuzzy logic.

E-mail : yck@gwnu.ac.kr

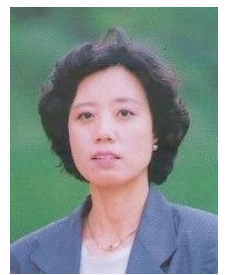

fuzzy logic.

\section{Jung Mi Ko}

She received the M.S and Ph.D. degrees in Department of Mathematics from Yonsei University, in 1983 and 1988, respectively. From 1988 to present, she is a professor in Department of Mathematics, GangneungWonju University. Her research interests are
E-mail: jmko@gwnu.ac.kr 